\title{
Correlation of Heat Shock Protein Expression to Gender Difference in Development of Stress-Induced Gastric Mucosal Injury in Rats
}

\author{
Taiji Takahashi ${ }^{1 *}$, Michiro Otaka ${ }^{1}$, Masaru Odashima ${ }^{3}$, Kumiko Tamaki $^{1}$, Makiko Takada ${ }^{1}$, \\ Yuko Izumi ${ }^{1}$, Tomoyoshi Shibuya ${ }^{1}$, Naoto Sakamoto ${ }^{1}$, Hideaki Itoh ${ }^{2}$ and Sumio Watanabe ${ }^{1}$ \\ ${ }^{1}$ Department of Gastroenterology, Juntendo University School of Medicine, Bunkyo-Ku, Tokyo 113-8421, Japan \\ ${ }^{2}$ Department of Life Science Faculty of Engineering and Resource Science, Akita University, \\ Akita-city, Akita 010-0852, Japan \\ ${ }^{3}$ Department of Gastroenterology, Yokote Municipal Hospital, Yokote-city, Akita 013-8602, Japan
}

Received 5 March, 2010; Accepted 19 March, 2010; Published online 26 May, 2010

\begin{abstract}
Summary Recent studies have indicated that heat shock proteins (HSPs), which function as molecular chaperones, play important roles in cellular responses to stress-related events. However, the gender difference in the expression of HSP in the gastric mucosa remains unclear. In order to understand the mechanism of gender difference in the prevalence or severity of gastric mucosal lesions, the expression level of HSP and the correlation of estrogen to HSP induction in the gastric mucosa were evaluated in this study. The basal expression levels of HSP60 and HSP90 in the gastric mucosa were significantly higher in females than those in males. The gastric ulcer index was significantly higher in male rats compared to female rats observed after $12 \mathrm{~h}$ water immersion stress exposure. At this time point, the expression levels of HSP60 and HSP90 in the gastric mucosa were significantly higher in females than those in males. An estrogen-treatment significantly induced the expression of HSP60, HSP70 and HSP90 in the gastric mucosa. Inversely, an ovariectomy dramatically reduced the expression of HSP60, HSP70 and HSP90 in the gastric mucosa. Our results suggested that estrogen might play an important role in gastric mucosal protection with the induction of gastric mucosal HSPs.
\end{abstract}

Key Words: heat shock protein, gender difference, gastric mucosal protection, estrogen, stress

\section{Introduction}

Many epidemiological investigations have shown that the prevalence of peptic ulcers is higher in men compared to that in women, although the mechanisms underlying this gender difference are not fully understood [1-3]. Numerous studies have reported the protective role of estrogen in the development of various diseases such as cardiovascular disease [4], osteoporosis [5], and cerebral damage [6]. Therefore, sex hormones have been considered as the modulating factors in these diseases. In contrast, reports regarding the protective

\footnotetext{
*To whom correspondence should be addressed

Tel: +81-3-3813-3111 Fax: +81-3-3813-8862

E-mail: ttakahas@juntendo.ac.jp
}

effects of sex hormones in the gastrointestinal tract are extremely limited $[7,8]$.

Previous studies showed that various stresses, such as heat (e.g. hyperthermia), toxins, and some heavy metals induce heat shock proteins (HSPs), which function as molecular chaperones, play important roles in cellular responses to stress-related events. HSPs have been classified into four families, HSP90, HSP70, HSP60, and the low molecular weight HSP family, based on their subunit molecular masses and structural homology. HSPs are known to be highly conserved and are ubiquitous proteins expressed by eukaryotic and prokaryotic cells $[9,10]$. Therefore, it is thought that HSPs have essential functions for the survival of cells and developmental process. Our recent studies have focused on the expression and 'chaperone' function 
a

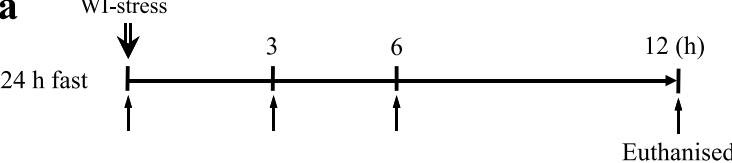

( $n=4$ at each time point)

b

Male
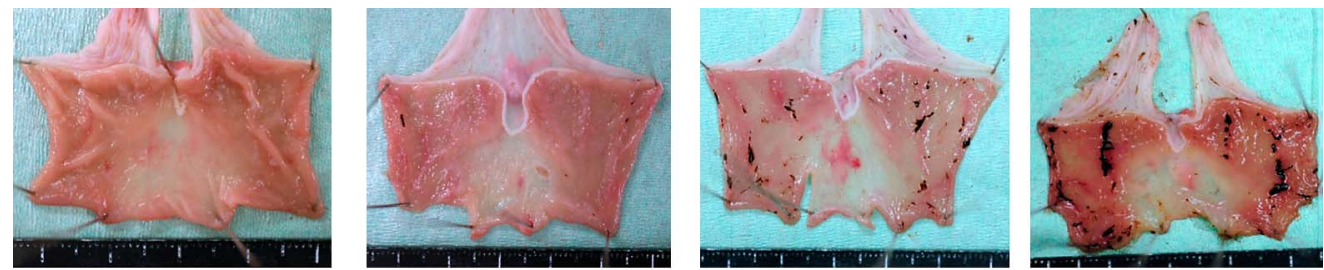

Female

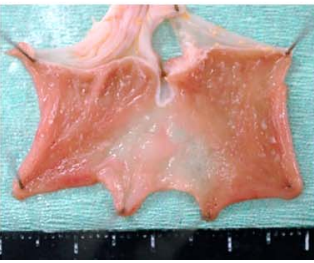

control

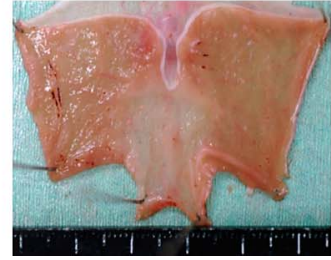

3h-WI-stress

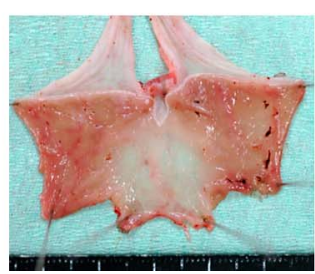

d ${ }_{\mathrm{HSP} 60}$

$\mathbf{e}$

c

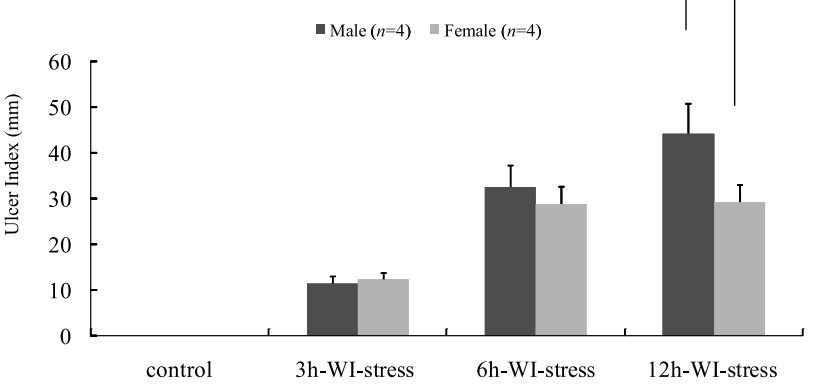

e $\quad$ HSP60

HSP70
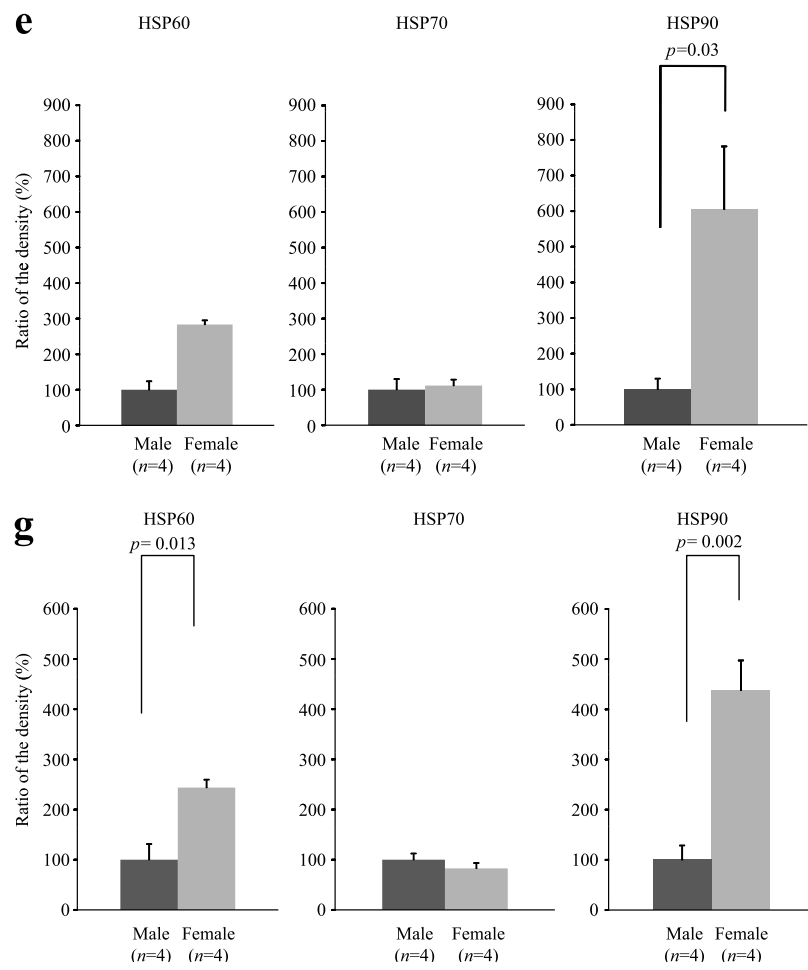

HSP70
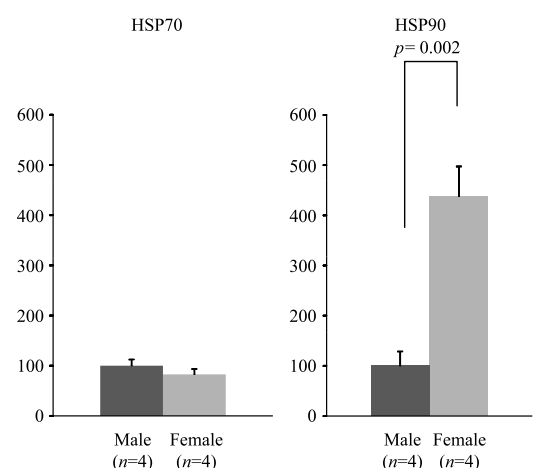

6h-WI-stress

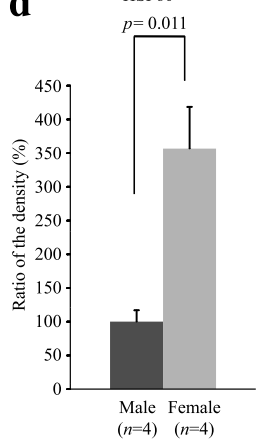

f ${ }_{\mathrm{HSP}} 6 \mathrm{O}$

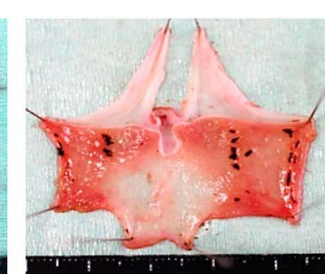

12h-WI-stress
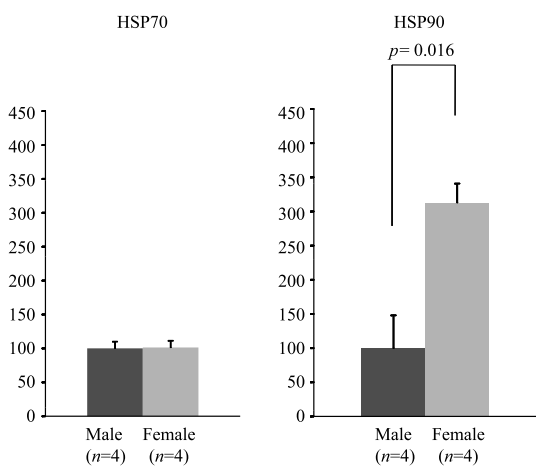

f

HSP70
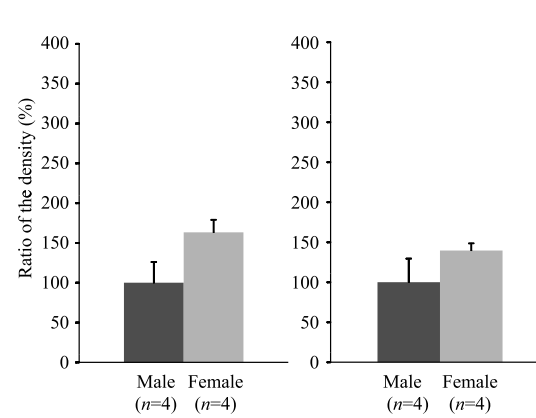

Vol. 47, No. 1, 2010 
Fig. 1. a: This figure shows the experimental protocol to study the gender difference in the ulcer index. Rats were euthanized before and 3, 6, or $12 \mathrm{~h}$ after the initiation of the WI-stress ( $n=4$ at each time point). WI-stress: water immersion stress; h: hour. b: Macroscopic findings showed that the gastric mucosal injuries after $12 \mathrm{~h}$ WI-stress exposure in male rats were more severe than those in female rats. WI-stress: water immersion stress; h: hour. c: This figure shows gender differences in the ulcer index. The ulcer index was significantly higher in male rats compared to that in female rats after the $12 \mathrm{~h}$ WI-stress (Bonferroni correction, $p=0.042$ ). No gender difference in the gastric ulcer index was observed at the $3 \mathrm{~h}$, or $6 \mathrm{~h}$ WI-stress exposure. Ulcer index: the total length of gastric mucosal erosions in mm; WI-stress: water immersion stress; h: hours; ${ }^{*} p<0.05$ was considered to be statistically significant. d: This figure shows gender differences in the basal expression of the HSPs. In the female rat gastric mucosa, the basal expression levels of HSP60 ( $t$ test, $p=0.011$ ) and HSP90 ( $t$ test, $p=0.016$ ) were significantly higher compared to those in male rats. There was no significant gender difference in the HSP70. Ratio of the density (\%): density (female HSP) / density (male HSP) $\times 100,{ }^{*} p<0.05$ was considered to be statistically significant. e: This figure shows the expression of HSPs after the $3 \mathrm{~h}$ WI-stress. There is no significant gender difference in the HSP70 and HSP60 levels. The expression of HSP90 was significantly higher in the females than in the males (Bonferroni correction, $p=0.03$ ). Ratio of the density (\%): density (female HSP) / density (male HSP) $\times 100,{ }^{*} p<0.05$ was considered to be statistically significant. f: This figure shows the expression of HSPs after the $6 \mathrm{~h}$ WI-stress. There is no significant gender difference in the HSP70 and HSP60 levels. The expression of HSP90 was significantly higher in the females than in the males (Bonferroni correction, $p=0.023$ ). Ratio of the density (\%): density (female HSP) / density (male HSP) $\times 100,{ }^{*} p<0.05$ was considered to be statistically significant. g: This figure shows the expression of HSPs after the $12 \mathrm{~h}$ WI-stress. The expression of HSP60 (Bonferroni correction, $p=0.013$ ) and HSP90 (Bonferroni correction, $p=0.002$ ) was significantly higher in the females than in the males, whereas there was no significant gender difference in the HSP70 level. Ratio of the density (\%): density (female HSP) / density (male HSP) $\times 100,{ }^{*} p<0.05$ was considered to be statistically significant.

of HSPs, chiefly their cytoprotective functions in digestive organs and cells. In the series of studies, we have demonstrated that HSPs are induced by several environmental stresses, including hyperthermia, neuropeptides, neuroamines, and various drugs in vivo. Some of the HSPs showed a cytoprotective function mediated by the chaperone function in the gastric mucosa. However, the relationship between the gender difference and the expression of HSPs in the gastric mucosa has not been studied.

In the present study, the expression of the HSPs levels and the effect of estrogen on the induction of HSPs in the gastric mucosa were evaluated to elucidate the mechanisms of gender difference in the prevalence or severity of gastric mucosal lesions.

\section{Materials and Methods}

\section{Animals}

This study was approved by the Animal Care Committee of the Juntendo University School of Medicine. Nine-weekold male and female Wistar rats (body weight: male 300$350 \mathrm{~g}$; female $250-300 \mathrm{~g}$ ) were fed a standard laboratory diet and water ad libitum and kept in cages (four rats were kept in a cage) in a temperature $\left(24 \pm 2^{\circ} \mathrm{C}\right)$-controlled room with a 12 h-dark-light ( 6 a.m. on, 6 p.m. off) cycle before and during the experiments. All of the experiments were performed using animals deprived of food for $24 \mathrm{~h}$, but with free access to water before the experiments including the control animals.
Water-immersion stress (WI-stress)-induced gastric mucosal lesions

Rats were placed in a restraint cage $[90 \times 240 \times 190 \mathrm{~mm}$ for four rats $(90 \times 60 \times 190 \mathrm{~mm} / \mathrm{rat})]$ and immersed vertically to the level of the xyphoid process from the foot in a water bath $(320 \times 420 \times 300 \mathrm{~mm})\left(23^{\circ} \mathrm{C}\right)[11,12]$. They were euthanized by stunning and cervical dislocation before and 3,6 , or $12 \mathrm{~h}(n=4$ at each time $)$ after the initiation of the WI-stress (Fig. 1a). The stomach was cut along the greater curvature and examined for mucosal lesions. The total length of each linear hemorrhagic erosion was measured by a stereoscopic microscope as the ulcer index (UI) (in $\mathrm{mm}$ ) [7] by two observers who were blind to the animal group.

\section{SDS-PAGE and Western blot analysis}

Each gastric mucosa was scraped off using two glass slides on ice and immediately used for further experiments. The gastric mucosa was homogenized with ice-cold lysis buffer containing $50 \mathrm{mM}$ Tris- $\mathrm{HCl}, \mathrm{pH} 8.0,150 \mathrm{mM}$ $\mathrm{NaCl}, 1 \mathrm{mM}$ ethylene diamine tetraacetic acid (EDTA), $1 \%$ TritonX-100, and Complete Mini according to the manufacturer's recommendation (EDTA-free, Protein inhibitor cocktail tablets, Mannheim, Germany). The homogenates were centrifuged at $17,400 \mathrm{~g}$ for $20 \mathrm{~min}$ at $4^{\circ} \mathrm{C}$, and the supernatant was stored at $-80^{\circ} \mathrm{C}$ until the Western blotting was performed. The protein concentrations were measured by a protein assay kit (Bio-Rad, Richmond, California) using bovine serum albumin as the standard. An equal total protein amount was used for the 9\% SDS-polyacrylamide gel electrophoresis [13]. Gels were stained with $0.1 \%$ Coomassie Brilliant Blue (R-250) in a mixture of $25 \%$ 
a
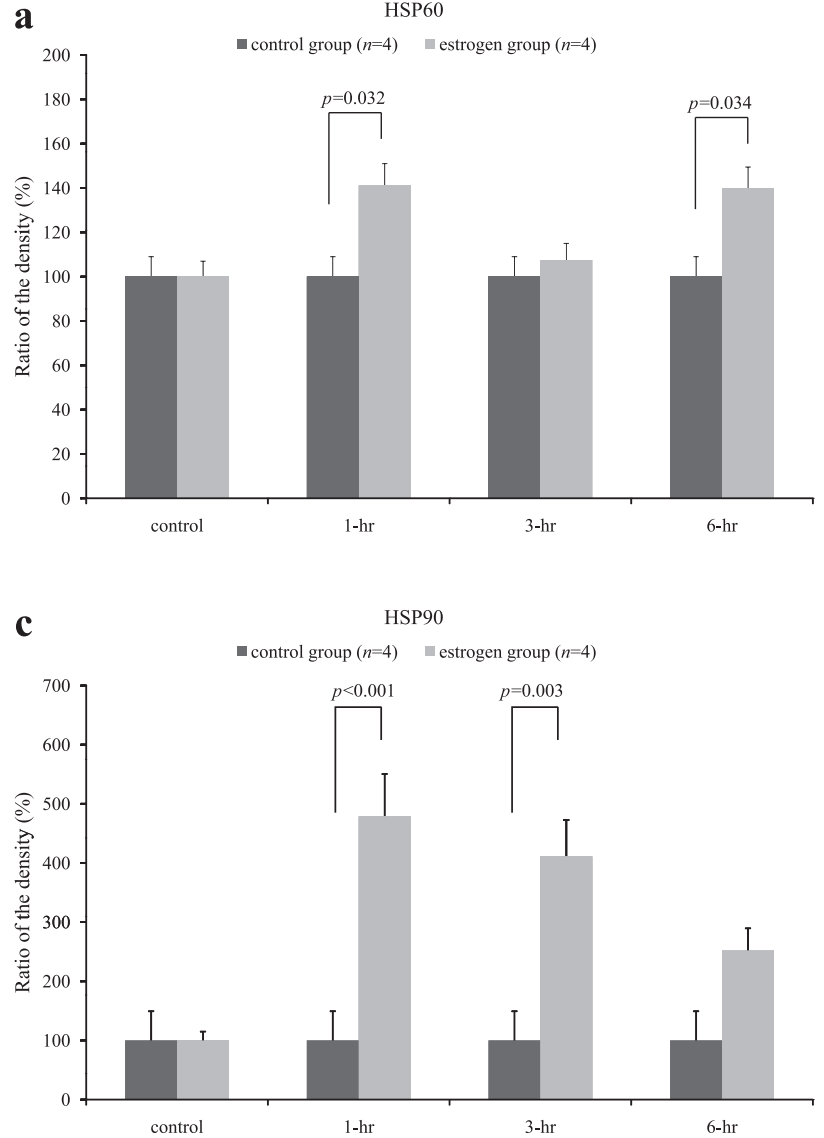

b
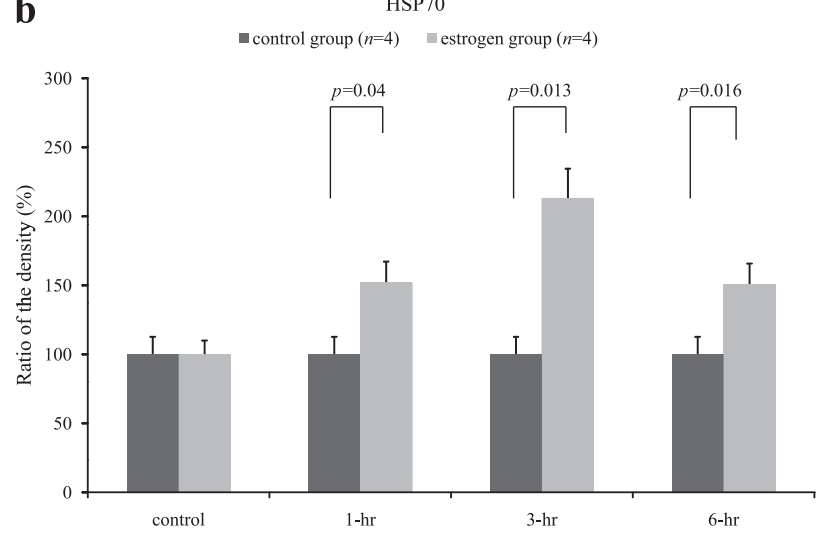

Fig. 2. In order to study the gender difference in the expression levels of the HSPs, we designed the experimental protocol to study the effect of estradiol on the male gastric mucosa. Each rat was euthanized before and $1 \mathrm{~h}, 3 \mathrm{~h}$, or $6 \mathrm{~h}$ after the intra-peritoneal injection of $10 \mathrm{mg} / \mathrm{kg}$ estradiol ( $n=4$ at each time point). In the control group, the rats were treated with the vehicle ( $n=4$ at each time point). a: The expression level of HSP60 was significantly higher in the estradiol-administered group (estrogen group) compared to the control group after the $1 \mathrm{~h}$ (Bonferroni correction, $p=0.032$ ) and $6 \mathrm{~h}$ (Bonferroni correction, $p=0.034)$. b: The expression level of HSP70 was significantly higher in the estradiol-administered group (estrogen group) compared to the control group after the $1 \mathrm{~h}$ (Bonferroni correction, $p=0.04$ ), $3 \mathrm{~h}$ (Bonferroni correction, $p=0.013$ ) and $6 \mathrm{~h}$ (Bonferroni correction, $p=0.016)$. $\mathrm{c}$ : The expression level of HSP90 was significantly higher in the estradiol-administered group (estrogen group) compared to the control group after the $1 \mathrm{~h}$ (Bonferroni correction, $p<0.001$ ), $3 \mathrm{~h}$ (Bonferroni correction, $p=0.003)$. E2: estradiol; $1 \mathrm{~h}: 1 \mathrm{~h}$ after intra-peritoneal injection of vehicle (DMSO/PBS, $0.5 \mathrm{ml})$ or estradiol (10 mg/kg); $3 \mathrm{~h}$ : $3 \mathrm{~h}$ after intra-peritoneal injection of vehicle (DMSO/PBS, $0.5 \mathrm{ml})$ or estradiol $(10 \mathrm{mg} / \mathrm{kg}) ; 6 \mathrm{~h}: 6 \mathrm{~h}$ after intra-peritoneal injection of vehicle (DMSO/PBS, $0.5 \mathrm{ml})$ or estradiol $(10 \mathrm{mg} / \mathrm{kg})$; Ratio of the density $(\%)=\operatorname{density}(\mathrm{E} 2 \mathrm{ip}) / \mathrm{density}$ $($ control $) \times 100,{ }^{*} p<0.05$ was considered to be statistically significant.

isopropyl alcohol- $10 \%$ acetic acid and destained with $10 \%$ isopropyl alcohol- $10 \%$ acetic acid.

The expression of each HSP was evaluated by a previously reported method [14]. Briefly, the samples $(25 \mu \mathrm{g}$ of protein/lane) were electrophoresed on 9\% SDSpolyacrylamide gels, electrophoretically transferred to a PVDF membrane $(0.45 \mu \mathrm{m}$ pore size; Amersham Pharmacia Biotech, Buckinghamshire, England), and processed as described by Towbin et al. [15]. The membrane was incubated with the rabbit anti-HSP60 antibody [16], rabbit antiHSP70 antibody [17], or rabbit anti-HSP90 antibody [18] and treated with horseradish peroxidase-conjugated anti- rabbit $\operatorname{IgG}(1: 1000)$. The stained bands were visualized with $0.02 \%$ 3,3-diaminobenzidine tetrahydrochloride containing $0.005 \% \mathrm{H}_{2} \mathrm{O}_{2}$. The intensity of the visualized band was quantified using Quantify One.4.0 software (Bio-Rad, Richmond, California). The ratio of the density of the stained band was calculated using the following formula as previously reported [19]. In Fig. 1d-g, Ratio of the density $(\%)=$ density $($ female HSP $) /$ density $($ male HSP $) \times 100$; in Fig. 2a-c, Ratio of the density $(\%)=$ density (esreadiol (E2) i.p.) / density (control) $\times 100$; in Fig. 3 , Ratio of the density $(\%)=$ density (ovariectomy) / density (sham operation) $\times 100$. 

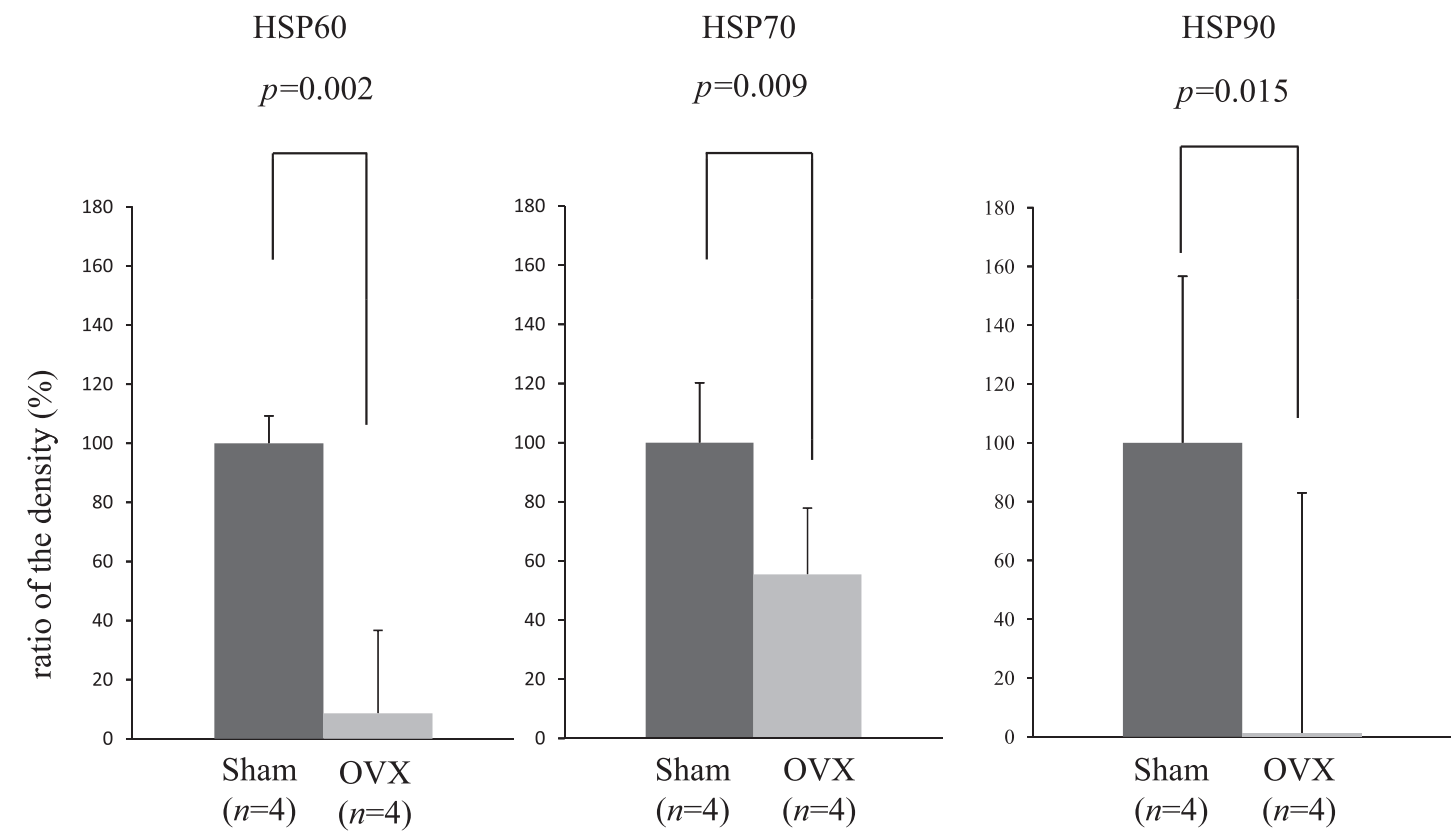

Fig. 3. We designed the experimental protocol to study the effect of an ovariectomy (OVX) on the HSP expression in the female rat gastric mucosa. Eight female rats were divided into two groups. The sham group was given a sham operation $(n=4)$. The ovariectomy group was subjected to a bilateral ovariectomy $(n=4)$. Each group was euthanized 2 weeks after the operation and the stomach of each rat was removed. The ovariectomy dramatically reduced the expression of HSP60 $(t$ test, $p=0.002)$, HSP70 ( $t$ test, $p=0.009$ ), and HSP90 ( $t$ test, $p=0.015$ ) compared to the sham-operated group. Ratio of the density $(\%)=$ density (ovariectomy) / density (sham operation) $\times 100 ;{ }^{*} p<0.05$ was considered to be statistically significant.

\section{Effect of E2 on gastric mucosal HSP expression}

In order to study the effect of the E2 injection on the gastric mucosal HSP expression, 20 male rats were divided into four groups, and the experimental protocol was designed as follows. In the estrogen group, each rat was euthanized 1,3 , or $6 \mathrm{~h}$ ( $n=4$ at each time point) after the end of the intra-peritoneal injection of $10 \mathrm{mg} / \mathrm{kg}$ of $\mathrm{E} 2$ (This is a supraphysiological dose; other studies also treated animals with $1 \mathrm{mg} / \mathrm{kg}-10 \mathrm{mg} / \mathrm{kg}$ of E2 [20,7] to study the effect of E2 on gastric ulceration with elevated serum levels of E2) (Cayman Chemical, Ann Arbor, Michigan) dissolved in a DMSO/PBS (DMSO: PBS = 1:4) solution $(0.2 \mathrm{mg} / \mathrm{ml})$. The animals were not given free access to food and water after the intra-peritoneal injection of E2. In the control group, the rats were treated with the vehicle. The stomach was then removed and the gastric mucosa of each rat was obtained.

In order to examine whether the stress-induced gastric mucosal lesions were reduced by the estrogenic administration, we designed the experimental protocol. Eight male rats were divided into two groups. The control group $(n=4)$ received an intra-peritoneal injection of $0.5 \mathrm{ml}$ DMSO/PBS (DMSO: $\mathrm{PBS}=1: 4$ ) as the vehicle. The estrogen group $(n=4)$ received an intra-peritoneal injection with $10 \mathrm{mg} / \mathrm{kg}$ of E2. DMSO/PBS and the E2 were received $1 \mathrm{~h}$ before the initiation of the $12 \mathrm{~h}$ WI-stress exposure.
Effect of ovariectomy on gastric mucosal HSP expression

Anesthesia was performed by inhalation anesthesia using isoflurane [21]. The female rats in the sham group $(n=4)$ underwent a sham operation. The female rats in the ovariectomy group $(n=4)$ were subjected to a bilateral ovariectomy by a ventral approach. Each group was euthanized 2 weeks after the operation and the stomach of each rat was then removed.

In order to study the effect of the E2 pretreatment on the ovariectomized female gastric mucosal protection, we designed the experimental protocol. Eight ovariectomized female rats were divided into a control group and an estrogen group. The control group $(n=4)$ received an intraperitoneal injection of $0.5 \mathrm{ml}$ DMSO/PBS (DMSO: PBS $=1: 4)$ as the vehicle. The estrogen group $(n=4)$ was treated with $10 \mathrm{mg} / \mathrm{kg}$ of E2 (i.p.). In both groups, the rats were euthanized after the initiation of a $12 \mathrm{~h}$ WI-stress.

\section{Data analysis}

All data were expressed as mean \pm SEM. A statistical analysis was performed using a post-hoc test (Bonferroni correction) after two-way ANOVA to identify differences between individual groups for multiple comparisons (Fig. 1c, e-g, and 2a-c). For unpaired variants, $t$ test was performed after $\mathrm{F}$ test (Fig. 1d, 3, 4 and 5); $p<0.05$ was considered to be statistically significant. 


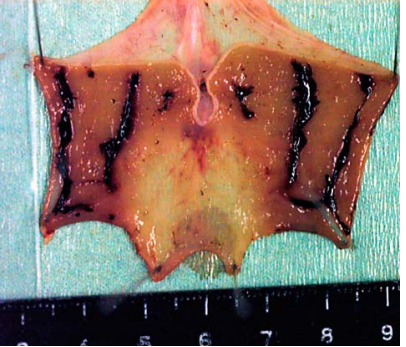

Control group

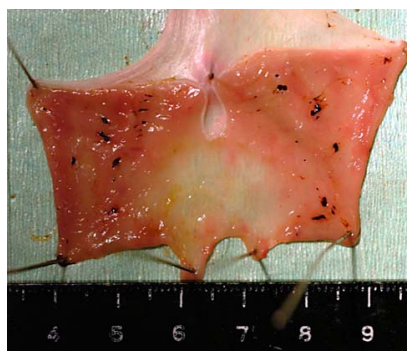

Estrogen group

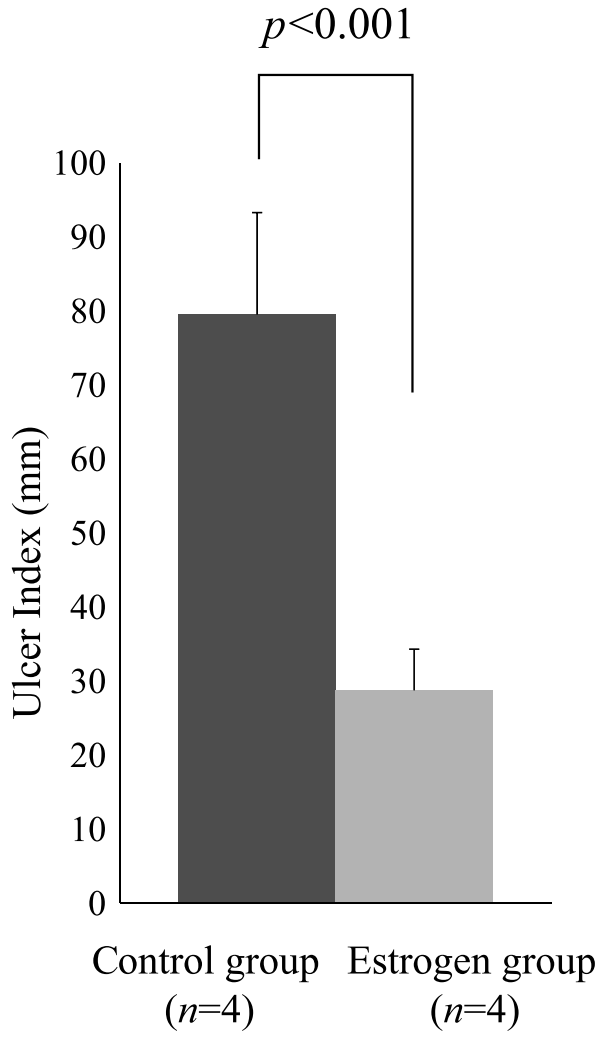

Fig. 4. In order to examine whether the stress-induced gastric mucosal lesions were reduced by the estrogenic administration, we designed the experimental protocol. Eight male rats were divided into two groups. The control group received an intraperitoneal injection of the vehicle $(n=4)$. The estrogen group received an intra-peritoneal injection of $10 \mathrm{mg} / \mathrm{kg}$ estradiol $(n=4)$. The vehicle and estradiol were administered $1 \mathrm{~h}$ before the initiation of the $12 \mathrm{~h}$ WI-stress. As shown in this figure, the administration of estradiol significantly reduced the severity of the stress-induced gastric mucosal lesions (UI) in the male rats ( $t$ test, ${ }^{*} p<0.001$ ). Control group, vehicle [DMSO/PBS (DMSO: PBS = 1:4)] 0.5 ml, i.p; Estrogen group, E2 $10 \mathrm{mg} / \mathrm{kg}$ i.p; Ulcer index: the total length of gastric mucosal erosions in $\mathrm{mm} ;{ }^{*} p<0.05$ was considered to be statistically significant.

\section{Results}

\section{Ulcer index and expression of HSPs after WI-stress}

There was a significant main effect of the time after WIstress [degree of freedom $(\mathrm{df})=1, \mathrm{~F}=102.05, p<0.0001$ ] such that the UI of the time after WI-stress in the male rats was significantly higher than that in the female rats. There was not a main effect of the sex difference in the UI. There was also a sex $\times$ time interaction $[\mathrm{df}=1, \mathrm{~F}=5.55$, $p=0.026]$ in which there was significant sex difference in the time after WI-stress of the UI (Fig. 1c). As shown in Fig. $1 \mathrm{~b}$ and $\mathrm{c}$, the UI after the $12 \mathrm{~h}$ WI-stress was significantly higher in the male rats when compared to that in the female rats (Bonferroni correction, $p=0.042$ ). There was no significant gender difference in the UI after the $3 \mathrm{~h}$ or $6 \mathrm{~h}$ WI-stress exposure. In the female rat gastric mucosa, the basal expression levels of HSP60 ( $t$ test, $p=0.0011$ ) and HSP90 ( $t$ test, $p=0.016$ ) were significantly higher compared to those in the males (Fig. 1d). As shown in Fig. 1e-g, there was a significant main effect of the sex difference in the HSP expression [HSP60, $\mathrm{df}=1, \mathrm{~F}=30.7$, $p<0.0001$; HSP90, $\mathrm{df}=1, \mathrm{~F}=34.6, p<0.0001]$ such that the HSP60 and HSP90 expression after WI-stress in the female rats was significantly higher than that in the male rats, and there was also a main effect of time after the WI-stress [HSP60, $\mathrm{df}=3, \mathrm{~F}=12.8, p<0.0001]$ such that the male rats after the WI-stress expressed less HSP60 than that in the female rats. There was also a sex $\times$ time interaction [HSP60, $\mathrm{df}=3, \mathrm{~F}=12.8, p<0.0001]$ in which the female rats after WI-stress significantly induced the expression of HSP60 in the gastric mucosa compared to that in the males. There was neither main effects of sex and time after the WI-stress nor a sex $\times$ time interaction on the expression of the gastric mucosal HSP70. The induction ability of the gastric mucosal HSP60 (Bonferroni correction, $p=0.013$ ) and HSP90 (Bonferroni correction, $p=0.002$ ) by the $12 \mathrm{~h}$ WI-stress was significantly higher in the females than in the males (Fig. 1g). On the other hand, there is no significant gender difference in the induction of the HSP70 level. Especially, the expression level of HSP90 in the female gastric mucosa 


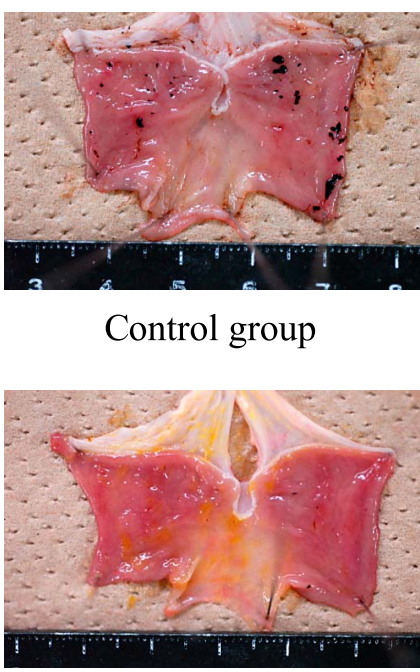

Estrogen group

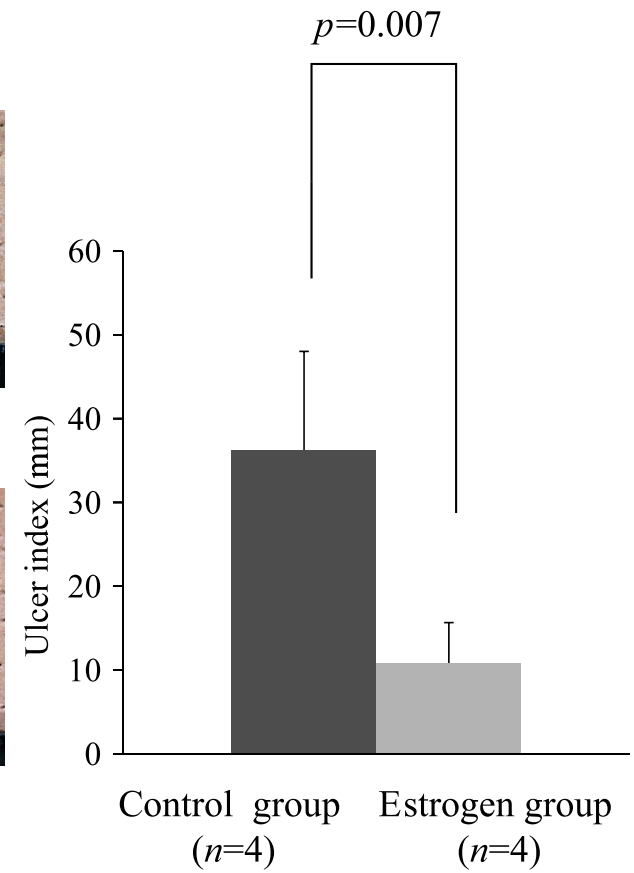

Fig. 5. In order to study the effect of the estradiol pretreatment on the ovariectomized female gastric mucosal protection, we designed the experimental protocol. Eight ovariectomized female rats were divided into a control group $(n=4)$ and an estrogen group $(n=4)$. The control group received an intra-peritoneal injection of the vehicle. The estrogen group was treated with $10 \mathrm{mg} / \mathrm{kg}$ estradiol. Each group of rats was euthanized after the initiation of the $12 \mathrm{~h} \mathrm{WI}$-stress. The treatment with estradiol significantly reduced the severity of the $12 \mathrm{~h}$ WI-stress-induced gastric mucosal lesions (UI) in the ovariectomized female rats $(t$ test, $p=0.007$ ). Control group: vehicle [DMSO/PBS (DMSO: PBS = 1:4) $0.5 \mathrm{ml}$ ] i.p.; Estrogen group: $\mathrm{E} 210 \mathrm{mg} / \mathrm{kg}$ i.p.; Ulcer index: the total length of gastric mucosal erosions in $\mathrm{mm}$; ${ }^{*} p<0.05$ was considered to be statistically significant.

increased from earlier time points and showed a higher level at all time points compared to in the male gastric mucosa (Bonferroni correction, $3 \mathrm{~h}$ WI-stress, $p=0.03 ; 6 \mathrm{~h}$ WIstress, $p=0.023,12 \mathrm{~h}$ WI-stress, $p=0.002$ ). There was no significant gender difference in the expression level of the HSP60 after the $3 \mathrm{~h}$ or $6 \mathrm{~h}$ WI-stress exposure.

In the male and female gastric mucosa, the time course of the actual expression levels of HSPs after WI-stress compared at each time point to the basal expression level (data not shown) showed similar results to the percentage data.

Effect of estrogen and ovariectomy on gastric HSP expression and mucosal protection

As shown in Fig. 2c, there was a significant main effect of the E2-treatment [HSP90, $\mathrm{df}=1, \mathrm{~F}=17.44, p=0.0005$ ], and there was also a main effect of time after the E2treatment [HSP90, $\mathrm{df}=1, \mathrm{~F}=7.85, p=0.011$ ] such that the control group expressed less HSP90 than the E2administered group. There was also an E2-treatment $\times$ time interaction [HSP90, $\mathrm{df}=1, \mathrm{~F}=7.87, p=0.011]$ in which the intra-peritoneal injection of E2 significantly induced the expression of HSP90 in the gastric mucosa compared to that in the untreated male control rats. The E2-treatment resulted in a significant increase in HSP60 and HSP70 in the gastric mucosa [HSP60, $\mathrm{df}=1, \mathrm{~F}=4.98, p=0.037 ; \mathrm{HSP} 70, \mathrm{df}=1$, $\mathrm{F}=16.80, p=0.0006]$ (Fig. 2a and b). There was neither a main effect of time after the E2-treatment nor an E2treatment $\times$ time interaction on the expression of the gastric mucosal HSP60 and HSP70. The expression levels of HSP60 (Bonferroni correction, $p=0.032$ ), HSP70 (Bonferroni correction, $p=0.04$ ), and HSP90 (Bonferroni correction, $p<0.001)$ were significantly higher in the E2-administered group (estrogen group) compared to the control group after $1 \mathrm{~h}$.

In order to examine whether the stress-induced gastric mucosal lesions were reduced by the estrogenic administration, the vehicle and E2 were administered $1 \mathrm{~h}$ before the initiation of the $12 \mathrm{~h}$ WI-stress. The administration of E2 significantly reduced the severity of the $12 \mathrm{~h}$ WI-stressinduced gastric mucosal lesions in male rats $(t$ test, $p<0.001)$ (Fig. 4).

As shown in Fig. 3, the ovariectomy dramatically reduced the expression of HSP60 ( $t$ test, $p=0.002)$, HSP70 ( $t$ test, $p=0.009$ ), and HSP90 ( $t$ test, $p=0.015$ ) compared to the sham-operated group. The treatment with E2 significantly reduced the severity of the $12 \mathrm{~h}$ WI-stress-induced gastric mucosal lesions in the ovariectomized female rats $(t$ test, $p=0.007)$ (Fig. 5). 


\section{Discussion}

Many recent studies have suggested that the synthesis of the HSPs is induced when the cells are exposed to sublethal stresses, such as elevated temperature, chemicals, infection, inflammation, or ischemia [16, 22, 23]. It has been reported that the heat shock response that induces HSPs is the most highly conserved genetic system known and exists in virtually every organism [24, 25]. HSPs are suggested to support the transportation, folding, and rearrangement of other proteins as a "molecular chaperon" $[9,10]$. A growing body of evidence has indicated that HSPs are produced in response to an initial sublethal stress to protect the organism from subsequent exposure to more severe stress [26]. Some series of studies, including ours [10,27], has focused on the cytoprotective functions of HSPs both in vitro and in vivo. In the gastrointestinal mucosa and the liver, many studies have shown that the HSP70 family has a cytoprotective function [28-30]. For example, zinc L-carnosine, which is one of the gastroprotectants, induced HSP70 in rat gastric mucosa [31]. In addition, gastroprotectants accelerated ulcer healing and resistance to ulcer recurrence in rat gastric mucosa [32].

On the other hand, numerous studies have reported the protective role of estrogen in the development of various diseases, such as cardiovascular disease [4], osteoporosis [5], cerebral damage [6], and peptic ulcers $[7,8,11]$. Although recent studies have suggested a link between estrogen and the HSP expression in the hypothalamus, uterus, skeletal muscle, heart, and neural cells [33-36], the association of the HSP expression with a gender difference in the development of gastric mucosal injury has not been investigated. This information led us investigate whether the severity of gastric mucosal lesion and the expression of the HSP levels in the gastric mucosa after stress exposure would differ between males and females. Also, the effect of the estrogen administration on the induction of the HSPs in the male and ovarectomized female rat gastric mucosa was analyzed.

In the present study, the expression of HSP70, which has been reported to be important for gastric mucosal protection $[28,30]$ in the gastric mucosa, did not show a significant gender difference before and after the WI-stress. On the other hand, the present study demonstrated that the expression of HSP90 and HSP60 showed a gender difference. Female rats were more resistant to the development of gastric ulceration by the WI-stress due to a higher expression level of gastric HSP90 and HSP60 compared to the male rats. These data could suggest that the gender difference in the development of stress-induced gastric mucosal injury in rats might be related to the expression levels of HSP90 and HSP60 in the gastric mucosa. Furthermore, the treatment of male rats with estrogen significantly increased the expression of HSP60, HSP70, and HSP90 and reduced the severity of the WI-stress-induced gastric mucosal lesions. In addition, an ovariectomy reduced the expression of HSP60, HSP70, and HSP90 and significantly increased the severity of the WI-stress-induced gastric mucosal lesions in the female rats. Inversely, when ovariectomized female rats were treated with estrogen, the severity of the stressinduced gastric mucosal lesions was reduced with the recovered levels of HSP60, HSP70, and HSP90 in the gastric mucosa. These observations suggested that the gender difference observed in the development of the stressinduced gastric mucosal injury in rats might be partly explained by the differences in the gastric mucosal levels of HSP90, HSP70, and HSP60 induced by estrogen. Although a gender difference in the basal expression level of HSP70 and its induction ability was not observed, the mechanism was unclear. The balance of sex hormones may regulate the HSP70 expression in the gastric mucosa.

It is well-known that HSP60 is present as a mitochondrial protein in mammalian cells and that it plays an important cytoprotective role under stress conditions in vitro. We have previously reported that experimentally induced acute pancreatitis was dramatically prevented by the pre-induction of HSP60 in rats $[16,37]$. In the gastric mucosal cells, HSP60 is induced in the cytoplasm and nuclei after the WIstress [38]. However, the function of HSP60 in the gastric mucosa has not been well understood. In the present study, the expression of HSP60 in the female gastric mucosa was higher than that in the male gastric mucosa before and after the WI-stress exposure. Both the female and male rats treated with estrogen were more resistant to the development of gastric ulceration by WI-stress with a higher expression level of HSP60. These findings might suggest that HSP60 is regulated by estrogen and correlated to the gastric mucosal protection.

HSP90 is known to act in the folding and maturation of steroid receptors [39]. Bharadwaj et al. reported that HSP90 regulates key steps in the heat shock transcription factor 1 (HSF1) activation-deactivation process [40]. Another recent report demonstrated that hypoxia-induced HSP90 $\alpha$ secretion required neither a change in the steady-state mRNA level nor in the promoter activity of HSP90 $\alpha$. More intriguingly, the topical application of HSP90 $\alpha$ accelerated wound healing in mice [41]. Recent studies have demonstrated that in the ventromedial hypothalamus (VMH) and uterus, the HSP90 expression was regulated by E2 [33, 42]. Taking these reports and our present data together, HSP90 is known to play an important role in the reduction of stress-induced gastric mucosal injury in females via an estrogen-HSP90 relationship with HSF1 activation.

\section{Conclusion}

Female rats were more resistant to the development of 
gastric mucosal injury by WI-stress. These phenomena were closely related to the mucosal levels of HSP90 and HSP60 in the gastric mucosa. Our results demonstrate for the first time that estrogen might play an important role in gastric mucosal protection inducing gastric mucosal HSPs. Our findings might at least partly explain one of the mechanisms of gender difference in gastric mucosal protection.

\section{References}

[1] Clark, D.H.: Peptic ulcer in women. Br. Med. J., 1, 12541257, 1953.

[2] Sawrey, W.L. and Long, D.H.: Strain and sex differences in ulceration in the rat. J. Comp. Physiol. Psychol., 55, 603605, 1962.

[3] de Martel, C. and Personnet, J.: Helicobacter pylori infection and gender: a meta analysis of population-based prevalence surveys. Dig. Dis. Sci., 51, 2292-2301, 2006.

[4] Kher, A., Wang, M., Tsai, B.M., Pitcher, J.M., Greenbaum, E.S., Nagy, R.D., Patel, K.M., Wairiuko, G.M., Markel, T.A., and Meldrum, D.R.: Sex differences in the myocardial inflammatory response to acute injury. Shock, 23, 1-10, 2005.

[5] Feng, W., Cui, Y., Song, C., Zhan, H., Wang, X., Li, Q., Cui, W., Guo, K., Maki, M., Hisha, H., Mori, T., and Ikehara, S.: Prevention of osteoporosis and hypogonadism by allogeneic ovarian transplantation in conjunction with intra-bone marrow-bone marrow transplantation. Transplantation, 84, 1459-1466, 2007.

[6] Kuiper, G.G., Shughrue, P.J., Merchenthaler, I., and Gustafsson, J.A.: The estrogen receptor beta subtype: a novel mediator of estrogen action in neuroendocrine systems. Front. Neuroendocrinol., 19, 253-286, 1998.

[7] Shimozawa, N., Okajima, K., Harada, N., Arai, M., Ishida, Y., Shimada, S., Kurihara, H., and Nakagata, N.: Contribution of sensory neurons to sex difference in the development of stress-induced gastric mucosal injury in mice. Gastroenterology, 131, 1826-1834, 2006.

[8] Machowska, A., Szlachcic, A., Pawlik, M., Brzozowski, T., Konturek, S.J., and Pawlik, W.W.: The role of female and male sex hormones in the healing process of preexisting lingual and gastric ulcerations. J. Physiol. Pharmacol., 55, 91-104, 2004.

[9] Hightower, L.E.: Heat shock, stress proteins, chaperones, and proteotoxicity. Cell, 66, 191-197, 1991.

[10] Otaka, M., Odashima, M., and Watanabe, S.: Role of heat shock proteins (molecular chaperones) in intestinal mucosal protection. Biochem. Biophys. Res. Commun., 348, 1-5, 2006.

[11] Takagi, K. and Okabe, S.: The effects of drugs on the production and recovery processes of the stress ulcer. Jpn. J. Pharmacol., 18, 9-18, 1968.

[12] Ohta, Y., Kaida, S., Chiba, S., Tada, M., Teruya, A., Imai, Y., and Kawanishi, M.: Involvement of oxidative stress in increases in the serum levels of various enzymes and components in rats with water-immersion restraint stress. J. Clin.
Biochem. Nutr., 45, 347-354, 2009.

[13] Laemmli, U.K.: Cleavage of structural proteins during the assembly of the head of bacteriophage T4. Nature, 227, 680$685,1970$.

[14] Otaka, M., Itoh, H., Kuwabara, T., Zeniya, A., Fujimori, S., Otani, S., Tashima, Y., and Masamune, O.: Induction of heat shock protein and prevention of caerulein-induced pancreatitis by water-immersion stress in rats. Int. J. Biochem., 26, 805-811, 1994.

[15] Towbin, H., Staehelin, T., and Gordon, J.: Electrophoretic transfer of proteins from polyacrylamide gels to nitrocellulose sheets: procedure and some applications. Proc. Natl. Acad. Sci. U.S.A., 76, 4350-4354, 1979.

[16] Otaka, M., Itoh, H., Kuwabara, T., Zeniya, A., Fujimori, S., Tashima, Y., and Masamune, O.: Induction of a $60-\mathrm{kDa}$ heat shock protein in rat pancreas by water-immersion stress. Int. J. Biochem., 25, 1769-1773, 1993.

[17] Wakui, H., Itoh, H., Tashima, Y., Kobayashi, R., Nakamoto, Y., and Miura, A.B.: Specific antibodies against the stressinducible $72-\mathrm{kDa}$ protein, a member of the heat-shock protein hsp70, in healthy human subjects. Int. J. Biochem., 23, 975-978, 1991.

[18] Itoh, H., Toyoshima, I., Mizunuma, H., Kobayashi, R., and Tashima, Y.: Three-step purification method and characterization of the bovine brain $90-\mathrm{kDa}$ heat shock protein. Arch. Biochem. Biophys., 282, 290-296, 1990.

[19] Otani, S., Otaka, M., Jin, M., Okuyama, A., Itoh, S., Iwabuchi, A., Sasahara, H., Itoh, H., Tashima, Y., and Masamune, O.: Effect of preinduction of heat shock proteins on acetic acid-induced colitis in rats. Dig. Dis. Sci, 42, 833846, 1997.

[20] Smith, A., Contreras, C., Ko, K.H., Chow, J., Dong, X., Tuo, B., Zhang, H.H., Chen, D.B., and Dong, H.: Gender-specific protection of estrogen against gastric acid-induced duodenal injury: stimulation of duodenal mucosal bicarbonate secretion. Endocrinology, 149, 4554-4566, 2008.

[21] Izumi, Y., Otaka, M., Takahashi, T., Takada, M., Shimada, Y., Asaoka, D., Nagahara, A., Itoh, H., and Watanabe, S.: Specific induction of a $72-\mathrm{kDa}$ heat shock protein protects esophageal mucosa from reflux esophagitis. Life Sci., 84, 517-522, 2009.

[22] Zatloukal, K., Sohar, R., Lackinger, E., and Denk, H.: Induction of heat shock proteins in short-term cultured hepatocytes derived from normal and chronically griseofulvin-treated mice. Hepatology, 8, 607-612, 1988.

[23] Emami, A., Schwartz, J.H., and Borkan, S.C.: Transient ischemia or heat stress induced a cytoprotectant protein in rat kidney. Am. J. Physiol., 260, F479-F485, 1991.

[24] Li, G.C., Li, L., Liu, R.Y., Rehman, M., and Lee, W.M.: Heat shock protein hsp70 protects cells from thermal stress even after deletion of its APT-binding domain. Proc. Natl. Acad. Sci. U.S.A., 89, 2036-2040, 1992.

[25] Higashi, T., Takechi, H., Uemura, Y., Kikuchi, H., and Nagata, K.: Differential induction of mRNA species encoding several classes of stress proteins following focal cerebral ischemia in rats. Brain Res., 650, 239-248, 1994.

[26] Li, G.C., Meyer, J.L., Mak, J.Y., and Hahn, G.M.: Heat- 
induced protection of mice against thermal death. Cancer Res., 43, 5758-5760, 1983.

[27] Nakamura, K., Rokutan, K., Marui, N., Aoike, A., and Kawai, K.: Induction of heat shock proteins and their implication in protection against ethanol-induced damage in cultured guinea pig gastric mucosal cells. Gastroenterology, 101, 161-166, 1991.

[28] Wada, I., Otaka, M., Jin, M., Odashima, M., Komatsu, K., Konishi, N., Matsuhashi, T., Horikawa, Y., Ohba, R., Itoh, H., and Watanabe, S.: Expression of HSP72 in the gastric mucosa is regulated by gastric acid in rats-correlation of HSP72 expression with mucosal protection. Biochem. Biophys. Res. Commun., 349, 611-618, 2006.

[29] Odashima, M., Otaka, M., Jin, M., Wada, I., Horikawa, Y., Matsuhashi, T., Ohba, R., Hatakeyama, N., Oyake, J., and Watanabe, S.: Zinc L-carnosine protects colonic mucosal injury through induction of heat shock protein 72 and suppression of NF-kappa B activation. Life Sci., 79, 22452250, 2006.

[30] Hirata, I., Naito, Y., Handa, O., Hayashi, N., Mizushima, K., Adachi, S., Omatsu, T., Okayama, T., Kishimoto, E., Ichikawa, H., Takagi, T., Kokura, S., Otaka, M., and Yoshikawa, T.: Heat-shock protein 70-overexpressing gastric epithelial cells are resistant to indomethacin-induced apoptosis. Digestion, 79, 243-250, 2009.

[31] Odashima, M., Otaka, M., Jin, M., Konishi, N., Sato, T., Kato, S., Matsuhashi, T., Nakamura, C., and Watanabe, S.: Induction of a $72-\mathrm{kDa}$ heat-shock protein in cultured rat gastric mucosal cells and rat gastric mucosa by zinc Lcarnosine. Dig. Dis. Sci., 47, 2799-2804, 2002.

[32] Oh, T.Y., Ahn, B.O., Jang, E.J., Park, J.S., Park, S.J., Baik, H.W., and Hahm, K.-B.: Accelerated ulcer healing and resistance to ulcer recurrence with gastroprotectants in rat model of Acetic Acid-induced Gastric Ulcer. J. Clin. Biochem. Nutr., 42, 204-214, 2008.

[33] Olazábal, U.E., Pfaff, D.W., and Mobbs, C.V.: Estrogenic regulation of heat shock protein $90 \mathrm{kDa}$ in rat ventromedial hypothalamus and uterus. Mol. Cell. Endocrinol., 84, 175183, 1992.
[34] Paroo, Z., Dipchand, E.S., and Noble, E.G.: Estrogen attenuates postexercise HSP70 expression in skeletal muscle. Am. J. Physiol. Cell Physiol., 282, C245-C251, 2002.

[35] Shinohara, T., Takahashi, N., Ooie, T., Ichinose, M., Hara, M., Yonemochi, H., Saikawa, T., and Yoshimatsu, H.: Estrogen inhibits hyperthermia-induced expression of heatshock protein 72 and cardioprotection against ischemia/ reperfusion injury in female rat heart. J. Mol. Cell Cardiol., 37, 1053-1061, 2004.

[36] Suuronen, T., Ojala, J., Hyttinen, J.M., Kaarniranta, K., Thornell, A., Kyrylenko, S., and Salminen, A.: Regulation of ER alpha signaling pathway in neuronal HN10 cells: role of protein acetylation and Hsp90. Neurochem. Res., 33, 17681775, 2008.

[37] Otaka, M., Okuyama, A., Otani, S., Jin, M., Itoh, S., Itoh, H., Iwabuchi, A., Sasahara, H., Odashima, M., Tashima, Y., and Masamune, O.: Differential induction of HSP60 and HSP72 by different stress situations in rats. Correlation with ceruleininduced pancreatitis. Dig. Dis. Sci., 42, 1473-1479, 1997.

[38] Fujimori, S., Otaka, M., Itoh, H., Kuwabara, T., Zeniya, A., Otani, S., Tashima, Y., and Masamune, O.: Induction of a $60-\mathrm{kDa}$ heat shock protein in rat gastric mucosa by restraint and water-immersion stress. J. Gastroenterol., 29, 544-546, 1994.

[39] Picard, D., Khursheed, B., Garabedian, M.J., Fortin, M.G., Lindquist, S., and Yamamoto, K.R.: Reduced levels of hsp90 compromise steroid receptor action in vivo. Nature, $\mathbf{3 4 8}$, 166-168, 1990.

[40] Bharadwaj, S., Ali, A., and Ovsenek, N.: Multiple components of the HSP90 chaperone complex function in regulation of heat shock factor 1 in vivo. Mol. Cell. Biol., 19, 80338041, 1999.

[41] Li, W., Li, Y., Guan, S., Fan, J., Cheng, C.F., Bright, A.M., Chinn, C., Chen, M., and Woodley, D.T.: Extracellular heat shock protein-90alpha: linking hypoxia to skin cell motility and wound healing. EMBO J., 26, 1221-1233, 2007.

[42] Ramachandran, C., Catelli, M.G., Schneider, W., and Shyamala, G.: Estrogenic regulation of uterine 90-kilodalton heat shock protein. Endocrinology, 123, 956-961, 1988. 